The Journal of Animal \& Plant Sciences, 30(5): 2020, Page: 1136-1144

ISSN (print): 1018-7081; ISSN (online): 2309-8694

\title{
SENSITIVITY AND SPECIFICITY OF ABDOMINAL FLUID VARIABLES AS A MARKER OF INTESTINAL ISCHEMIA IN DONKEYS (EQUUS ASINUS)
}

\author{
H. M. M. Ibrahim ${ }^{a^{*}}$, S. M. Shoieb ${ }^{\text {b }, ~ M . ~ R . ~ E l-A s h k e r ~}{ }^{\mathrm{a}}$ and K. S. Abouelnasr ${ }^{\mathrm{c}}$ \\ ${ }^{a}$ Department of Internal Medicine, Infectious and Fish Diseases, Faculty of Veterinary Medicine, Mansoura University, \\ Mansoura 35516, Egypt. \\ ${ }^{\mathrm{b}}$ Veterinary Teaching Hospital, Faculty of Veterinary Medicine, Mansoura University, Mansoura 35516, Egypt. \\ ${ }^{\mathrm{c}}$ Department of Surgery, Anesthesiology and Radiology, Faculty of Veterinary Medicine, Mansoura University, \\ Mansoura 35516, Egypt. \\ *Correspondence Author's E-mail: hussam-habosha@mans.edu.eg
}

\begin{abstract}
The current study aimed to assess the cellular profile and certain biochemical parameters of the peritoneal fluid, highlighting their diagnostic sensitivity and specificity as biomarkers for early diagnosis of intestinal ischemia in donkeys. For this purpose, peritoneal fluid samples were collected from 30 donkeys (Equus asinus) with intestinal obstruction and 10 randomly selected clinically healthy donkeys (Equus asinus) (control group) for cytological examination and biochemical analysis of the activity of alkaline phosphatase and lipase, and level of lactate, nitric oxide, and cardiac troponin I. In comparison with control group, the count of total nucleated cells, neutrophils, lymphocytes, and monocytes increased significantly $(\mathrm{P}<0.05)$. The cellular profile provoked $100 \%$ diagnostic sensitivity and specificity. Furthermore, the activity of alkaline phosphatase and lipase as well as the level of lactate, nitric oxide and cardiac troponin I in peritoneal fluid were greatly $(\mathrm{P}<0.05)$ increased in comparison with control group. Peritoneal fluid biochemical parameters elicited variable diagnostic sensitivity; alkaline phosphatase activity (90\%), lactate level (90\%), lipase activity (80\%), nitric oxide level (80\%), and cardiac troponin I level $(90 \%)$, with specificity (100\%) for each. Multivariate logistic regression analysis of peritoneal fluid variables demonstrated that increased total nucleated cells count, increased activity of both alkaline phosphatase and lipase, and increased lactate level are important factors for predicting intestinal obstruction and ischemia in the studied donkeys. The present study demonstrated that the changes in the cellular profile of the peritoneal fluid as well as biochemical analysis of the activity of both alkaline phosphatase and lipase, and level of lactate, nitric oxide, and cardiac troponin I in the peritoneal fluid are sensitive and specific predictors to assist in the early diagnosis of intestinal obstruction in donkeys (Equus asinus) with obstructive colic. The recorded alterations in the peritoneal fluid variables in donkeys (Equus asinus) may be used as a model for intestinal obstruction and ischemia in horses as well as other animal species.
\end{abstract}

Keywords: Obstructive colic; Intestinal ischemia; Peritoneal fluid; Donkeys (Equus asinus).

https://doi.org/10.36899/JAPS.2020.5.0130

Published online June 25, 2020

\section{INTRODUCTION}

Colic, an emergent condition encountered in equine practice, is a main cause of morbidity and mortality among horses and requires surgical intervention to resolve the main problem (Tinker et al., 1997). Approximately $50 \%$ of horses admitted in equine colic clinics required a rapid and emergent abdominal surgery (Hardy, 2004). The severity of colic case is ranged from temporary self-recovered spasmodic colic to severe strangulating obstruction with multi-organ failure and subsequent circulatory collapse (Archer and Proudman, 2006).

Acute circulatory failure secondary to intestinal ischemia and infarction is the leading cause of death as a direct result of equine colic. Therefore, quick and accurate detection of the bowel ischemic segment is necessary to prevent the complications with increasing the patient survival rate (Latson et al., 2005). Yet, there are several diagnostic tests for evaluation of horses with colic, and such tests vary in their costs, available facilities and experience level required to complete its techniques and interpreting the outcomes (Curtis et al., 2015).

Alkaline phosphatase, an enzyme present in intestinal mucosa, can be used as an indicator for strangulating versus non-strangulating intestinal lesions. Following experimental ileal ischemia, alkaline phosphatase activity in peritoneal fluid was significantly increased (Saulez et al., 2004). Moreover, horses with surgical colic are unique in having blood-stained peritoneal fluid with a high alkaline phosphatase activity. Furthermore, alkaline phosphatase activity is significantly different between survived horses and those that died, but not between horses treated surgically and those treated conservatively for the duration of colic (Kaya and Iben, 2009). 
Lipase, an enzyme catalyzes the breakdown of triglycerides, found not only in pancreatic acinar cells, but also in the heart, lungs, leukocytes and gastrointestinal tract, including the esophagus, duodenum, stomach, liver and colon (Tietz and Shuey, 1993; Lott and Lu, 1991). Therefore, serum lipase level may be mildly elevated in intra-abdominal diseases arising from the stomach, bowel and hepato-biliary tract (Hameed et al., 2015).

Lactate, the glycolysis end product, is usually increased because of poor tissue perfusion and anaerobic glycolysis that occurs in cases of shock (De Backer, 2003). In intestinal ischemia, the cell membrane permeability is deleteriously affected, with intracellular by-products, including the release of lactate into the peritoneal cavity (Liao et al., 1995).

Nitric oxide, a labile gas produced from the dietary amino acid L-arginine via nitric oxide synthase, exerts either cytoprotective or cytotoxic effects during intestinal ischemia and reperfusion (Moncada and Higgs, 1993; Beckman and Crow, 1993; Beckman and Koppenol, 1996). Endothelium nitric oxide synthasederived nitric oxide facilitates microvascular patency, inhibits platelet adhesion and aggregation, decreases neutrophil adhesion, and stabilizes mast cells, and thereby preserving the mucosal barrier function (Beckman and Crow, 1993; Mirza et al., 1999). Thus, nitric oxide synthesis is essential for the maintenance of both intestinal mucosal and microvascular barrier integrity of feline small intestine, which is damaged by ischemia and reperfusion (Kubes et al., 1992; Kubes, 1993). Furthermore, early administration of nitric oxide donors or L-arginine in the ischemia-reperfusion process decreases both mucosal and microvascular injury, protecting their function (Kanwar et al., 1994).

Earlier studies described the usefulness of estimating the peritoneal fluid $\mathrm{pH}$, glucose concentration, and activity of both alkaline phosphatase and lactate dehydrogenase as potential indicators of intestinal ischemia and peritonitis in horses (Latson et al., 2005; Van Hoogmoed et al., 1999; Yamout et al., 2011). However, there are no reported data on the significance of measuring the activity of lipase enzyme, and level of both nitric oxide and cardiac troponin I in peritoneal fluid in equine with obstructive colic. Thus, assessment of these biochemical markers in the peritoneal fluid may provide a clue for early detection of ischemic bowel injury in such species. In the current study, the donkey (Equus asinus), a domesticated member of the horse family, Equidae, was used as a model of equine species. In this article, the cellular profile and certain biochemical parameters, including the activity of alkaline phosphatase and lipase, and level of lactate, nitric oxide, and cardiac troponin I were evaluated in peritoneal fluid in donkey with obstructive colic, with a focus on their diagnostic sensitivity and specificity as biomarkers for the early diagnosis of intestinal ischemia in such animal species.

\section{MATERIALS AND METHODS}

Animals: The present study was conducted on 30 donkeys (Equus asinus) admitted to Veterinary Teaching Hospital, Mansoura University, Mansoura, Egypt, for colic of intestinal origin between May, 2017 and December, 2018. Their ages ranged between 3 and 10 years, and their body weight ranged between 150 and 280 $\mathrm{Kg}$. In addition, ten clinically healthy donkeys (Equus asinus) were randomly selected within the same age as a control group for this study. Organization and national rules for the utilization and care of the animals were taken as indicated by the Animal Welfare and Ethical Committee, Faculty of Veterinary Medicine, Mansoura University, Mansoura, Egypt, code No. R/9.

Clinical examination: All data related to the case history, and clinical findings of each donkey were recorded. A thorough history was taken, including age, sex, recent activity, feeding program, parasite control, fecal quality and when it was last passed, and any history of colic. Likewise, any changes in management practice, including feeding regimen, pregnancy, or medical treatments were recorded. The owners had also been asked about the time elapsed since onset of clinical signs of abdominal pain and any drugs given to the donkey, their amount, and how it is responding to analgesia. All studied donkeys were initially examined, including inspection of abdominal silhouette, abdominal auscultation, rectal examination, nasogastric intubation, abdominal paracentesis, and abdominal ultrasonography. A special concern was given to rectal temperature, heart rate, respiratory rate, mucous membranes, peripheral perfusion, appetite, severity of abdominal pain, motility of both cecum and colon, and defecation pattern, according to the standard clinical examination methods (Constable et al., 2017).

Ultrasonographic Examination: Transcutaneous ultrasonographic examination was conducted using a high-frequency linear $(6-11 \mathrm{MHz})$ array transducer (CHISON Digital Color Doppler Ultrasound system, iVis 60 EXPERT VET, CHISON Medical Imaging Co., Ltd., China) to examine the intestinal peristalsis. All ultrasound imaging procedures were performed using the standard protocol described previously by Gomaa et al. (2013); Ibrahim et al. (2013).

Peritoneal Fluid sampling and analysis: Peritoneal fluid samples were aseptically obtained from each standing donkey under investigation. A small stab incision was made directly to the right of midline using scalpel blade after local anesthesia, and then a sterile teat cannula was inserted (Latson et al., 2005; Constable et 
al., 2017) to collect two peritoneal fluid samples from each donkey. The first peritoneal fluid sample was collected in an ethylenediaminetetraacetic acid (EDTA) tube and immediately evaluated for color, turbidity, and cytological picture (total and differential cell counts) using standard method (Juzwiak et al., 1991; Hanson et al., 1992). The second peritoneal fluid sample was collected in a plain tube and immediately centrifuged $(1,500 \mathrm{x} \mathrm{g}, 10 \mathrm{~min})$, separated, and stored at $-20^{\circ} \mathrm{C}$ until biochemical analysis. All selected biochemical variables were quantified spectrophotometerically (Photometer 5010, Germany), using commercial test kits according to standard protocol of supplier; alkaline phosphatase activity (ELITech Group Clinical Systems Products, Russia), lactate level (Spectrum Diagnostics, Germany), lipase activity (Quimica Clinica Applicada, Spain), nitric oxide level (Bio-diagnostic, Egypt), and cardiac troponin I level (Siemens healthcare diagnostics products ltd, United Kingdom).

Statistical Analysis: Data analysis was performed using a statistical software program (SPSS for windows Version 21, SPSS Inc., Chicago, USA). For testing the normal distribution of the data, Kolmogrov-Smirnov test was used. The data were normally distributed; therefore, they were expressed as mean and standard deviation (SD). Independent sample t-test was used to determine the difference between diseased group and control group. ROC curve, sensitivity (\%), specificity (\%), accuracy (\%), and cut-off point for both the cytological picture and certain biochemical parameters of peritoneal fluid were performed. To examine the predictors for diagnosis of intestinal ischemia in donkeys, univariate logistic regression analysis model was performed using a constructed model from identified variables (cellular profile and certain biochemical parameters of the peritoneal fluid) by our investigation. Variables with significant association at $\mathrm{P}<0.05$ (two-sided) were further subjected to multivariate logistic regression model, providing a definition of the final parameters that early predict the intestinal ischemia in the studied donkeys. Regression coefficient (B), standard error (S.E.), Wald, P-value, odds ratio (OR), and a 95\% confidence interval $(95 \% \mathrm{CI})$ were recorded for each variable. In all statistical analyses, the results were considered significant at $\mathrm{P}<0.05$.

\section{RESULTS}

Clinically, both the heart rate and the respiratory rate were increased in the studied donkeys with intestinal obstruction compared with control group without alteration in the rectal temperature. Donkeys are very stoical, and therefore did not show the obvious clinical signs of colic that are seen in horses. Close monitoring of the studied donkeys with obstructive colic revealed that donkeys' demeanour and bowel habits were very helpful in identifying when they become unwell. The observed clinical signs in the donkeys with intestinal obstructive lesion were dullness, poor response to external stimuli, increased respiratory and heart rates, congested mucosa, anorexia, shivering, muscle fasciculation, excessive sweating, pawing, sternal recumbency, abdominal distension, and absence of both peristalsis and defecation. Rectal palpation revealed the presence of distended intestinal loops, and the rectum seemed sticky to touch and empty except for mucous and blood. At the terminal stage, the signs of hypovolemia as well as toxic shock with or without intestinal rupture and per-acute diffuse peritonitis appeared, but the pain did not worsen. There was no any fluid reflux on passage of a nasogastric tube in all studied donkeys with intestinal obstructive lesion.

Abdominal ultrasonography provided an indication about intestinal peristalsis, general condition of the intestine, and the amount of fluid in the abdominal cavity. In the studied donkeys with intestinal ischemia, the intestinal peristalsis was absent, the intestine appeared as several distended loops adjacent to each other with a thickened and edematous wall, and the amount of the fluid in the cranial abdomen increased.

The peritoneal fluid samples appeared turbid and tinged with blood in the studied donkeys with obstructive colic. There was a significant $(\mathrm{P}<0.05)$ increase in the count of total nucleated cells, neutrophils, lymphocytes, and monocytes in the studied donkeys with intestinal obstruction compared to the control group (Table 1). Besides, the cytological picture of peritoneal fluid had a diagnostic sensitivity of $100 \%$ and a specificity of $100 \%$ (Tables 2).

Univariate logistic regression analysis model of the cellular profile of peritoneal fluid showed that the increased count of each of the total nucleated cells, neutrophils, lymphocytes, and monocytes was a significant predictor of intestinal ischemia in the examined donkeys (Table 3). However, multivariate logistic regression analysis model showed that the increased total nucleated cells count in peritoneal fluid appeared to be an independent predictor of intestinal ischemia $(\mathrm{P}=0.001 ; \mathrm{OR}=0.028 ; 95 \% \mathrm{CI}=0.004-$ 0.196) (Table 4).

There was a significant $(\mathrm{P}<0.05)$ increase in the activity of both alkaline phosphatase and lipase as well as in the level of lactate, nitric oxide and cardiac troponin I in peritoneal fluid in donkeys with intestinal obstruction when compared with control group (Table 5). Moreover, the activity alkaline phosphatase, and the level of both lactate and cardiac troponin I in peritoneal fluid had a diagnostic sensitivity of $90 \%$ and a specificity of $100 \%$ for each of them. Besides, lipase activity and nitric oxide level in peritoneal fluid had a diagnostic sensitivity of 80 $\%$ and a specificity of $100 \%$ for each of them (Table 6 ). 
Univariate logistic regression analysis model of the selected biochemical parameters in peritoneal fluid indicated that the increased activity of both alkaline phosphatase and lipase and the increased level of lactate, nitric oxide, and cardiac troponin I in the peritoneal fluid were significant predictors of the intestinal ischemia in the studied donkeys (Table 7). However, multivariate logistic regression analysis model showed that the increased alkaline phosphatase activity in peritoneal fluid was a strong predictor of intestinal ischemia $(\mathrm{P}=0.034$; $\mathrm{OR}=0.041 ; 95 \% \mathrm{CI}=0.002-0.902)$. Likewise, the increased lipase activity in peritoneal fluid was a significant predictor of intestinal ischemia $(\mathrm{P}=0.030$; $\mathrm{OR}=0.056 ; 95 \% \mathrm{CI}=0.003-0.988)$. As well, the increased lactate level in peritoneal fluid was a substantial predictor of intestinal ischemia $(\mathrm{P}=0.018$; $\mathrm{OR}=0.030 ; 95 \% \mathrm{CI}=0.002-0.543)($ Table 8$)$.

Table 1. Peritoneal fluid cytological picture (Mean \pm SD) in clinically healthy donkeys and in those with obstructive colic.

\begin{tabular}{|c|c|c|c|c|}
\hline & 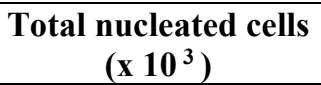 & $\begin{array}{l}\text { Neutrophils } \\
\left(\times 1^{3}\right)\end{array}$ & $\begin{array}{l}\text { Lymphocytes } \\
\left.\left(x^{10}\right)^{3}\right)\end{array}$ & $\begin{array}{c}\text { Monocytes } \\
\left(x \mathbf{1 0}^{3}\right)\end{array}$ \\
\hline Control $(n=10)$ & $1.05 \pm 0.05$ & $0.69 \pm 0.34$ & $0.32 \pm 0.30$ & $0.07 \pm 0.01$ \\
\hline Obstructive colic $(n=30)$ & $10.39 \pm 0.05^{*}$ & $5.39 \pm 1.49^{*}$ & $4.63 \pm 0.28^{*}$ & $0.37 \pm 0.02^{*}$ \\
\hline
\end{tabular}

${ }^{*}$ : Variables with different superscript in the same column are significantly different at $\mathrm{P}<0.05$.

Table 2. Sensitivity and specificity of analyzing the cytological picture of the peritoneal fluid in donkeys with obstructive colic.

\begin{tabular}{lcccc}
\hline & Total nucleated cells & Neutrophils & Lymphocytes & Monocytes \\
\hline AUC & 1.000 & 1.000 & 1.000 & 1.000 \\
Cut-off point $\left(\mathbf{x ~ 1 0}{ }^{3}\right)$ & 1.1 & 1.03 & 0.6 & 0.03 \\
Sensitivity (\%) & 100 & 100 & 100 & 100 \\
Specificity (\%) & 100 & 100 & 100 & 100 \\
95 \% confidence interval & $3.49-7.97$ & $1.95-4.27$ & $1.56-3.59$ & $0.10-0.26$ \\
Significance level P (Area=0.5) & 0.001 & 0.001 & 0.001 & 0.001 \\
\hline
\end{tabular}

Table 3. Univariate logistic regression model of the cytological picture of the peritoneal fluid in donkeys with obstructive colic.

\begin{tabular}{|c|c|c|c|c|c|c|}
\hline Variables & B & S.E. & Wald & $\mathbf{P}$ & OR & $95 \% \mathrm{CI}$ \\
\hline Total nucleated cells $\left(x \mathbf{1 0}^{3}\right)$ & 2.603 & 0.733 & 12.614 & 0.001 & 13.500 & $3.210-56.770$ \\
\hline Neutrophil $\left(\begin{array}{l}x \\
\left.10^{3}\right)\end{array}\right.$ & 2.485 & 0.736 & 11.400 & 0.001 & 12.000 & $2.836-50.775$ \\
\hline Lymphocytes $\left(x 1^{3}\right)$ & 2.398 & 0.739 & 10.541 & 0.001 & 11.000 & $2.587-46.779$ \\
\hline Monocytes $\left(x \mathbf{1 0}^{3}\right)$ & 2.351 & 0.740 & 10.096 & 0.001 & 10.500 & $2.462-44.781$ \\
\hline
\end{tabular}

Table 4. Multivariate logistic regression model of the cytological picture of the peritoneal fluid in donkeys with obstructive colic.

\begin{tabular}{lcccccc}
\hline Variables & B & S.E. & Wald & P & OR & 95 \% CI \\
\hline Total nucleated cells $\left(\mathbf{x ~ 1 0}^{\mathbf{3}}\right)$ & -3.584 & 0.998 & 12.901 & 0.001 & 0.028 & $0.004-0.196$ \\
Constant & 2.603 & 0.733 & 12.614 & 0.001 & & \\
\hline
\end{tabular}

Table 5. Activity of both alkaline phosphatase and lipase, and level of lactate, nitric oxide, and cardiac troponin I (Mean \pm SD) in the peritoneal fluid in clinically healthy donkeys and in those with obstructive colic.

\begin{tabular}{|c|c|c|c|c|c|}
\hline & $\begin{array}{c}\text { Alkaline phosphatase } \\
(\mathrm{U} / \mathrm{L})\end{array}$ & $\begin{array}{c}\text { Lipase } \\
\text { (U/L) }\end{array}$ & $\begin{array}{c}\text { Lactate } \\
(\mathrm{mmol} / \mathrm{L})\end{array}$ & $\begin{array}{c}\text { Nitric oxide } \\
(\mathrm{U} / \mathrm{mL})\end{array}$ & $\begin{array}{c}\text { Cardiac troponin I } \\
(\mathrm{ng} / \mathrm{mL})\end{array}$ \\
\hline Con & $15.08 \pm 0.75$ & $58.9 \pm 11.5$ & $3.00 \pm 0.23$ & $2.46 \pm 0.31$ & $0.02 \pm 0.01$ \\
\hline Obstructive colic $(n=30)$ & $19.70 \pm 2.26^{*}$ & $85.60 \pm 9.08^{*}$ & $9.69 \pm 2.8^{*}$ & $4.99 \pm 0.45^{*}$ & $0.05 \pm 0.01^{*}$ \\
\hline
\end{tabular}

${ }^{*}$ : Variables with different superscript in the same column are significantly different at $\mathrm{P}<0.05$. 
Table 6. Sensitivity and specificity of analyzing the activity of both alkaline phosphatase and lipase, and the level of lactate, nitric oxide, and cardiac troponin I in peritoneal fluid in donkeys with obstructive colic.

\begin{tabular}{lccccc}
\hline & $\begin{array}{c}\text { Alkaline Phosphatase } \\
(\mathbf{U} / \mathbf{L})\end{array}$ & $\begin{array}{c}\text { Lipase } \\
(\mathbf{U} / \mathbf{L})\end{array}$ & $\begin{array}{c}\text { Lactate } \\
(\mathbf{m m o l} / \mathbf{L})\end{array}$ & $\begin{array}{c}\text { Nitric oxide } \\
(\mathbf{U} / \mathbf{m L})\end{array}$ & $\begin{array}{c}\text { Cardiac troponin I } \\
(\mathbf{n g} / \mathbf{m L})\end{array}$ \\
\hline AUC & 0.965 & 0.980 & 0.970 & 0.935 & 0.905 \\
Cut-off point & 15.83 & 70.4 & 3.23 & 2.77 & 0.03 \\
Sensitivity (\%) & 90 & 80 & 90 & 80 & 90 \\
Specificity (\%) & 100 & 100 & 100 & 100 & 100 \\
$\mathbf{9 5 \%}$ confidence interval & $0.888-1.042$ & $0.929-1.031$ & $0.902-1.038$ & $0.833-1.037$ & $0.768-1.042$ \\
Significance level P (Area=0.5) & 0.001 & 0.001 & 0.001 & 0.001 & 0.002 \\
\hline
\end{tabular}

Table 7. Univariate logistic regression model of the analysis of the activity of both alkaline phosphatase and lipase, and the level of lactate, nitric oxide, and cardiac troponin $I$ in peritoneal fluid in donkeys with obstructive colic.

\begin{tabular}{lcccccc}
\hline Variables & B & S.E. & Wald & P & OR & 95 \% CI \\
\hline Alkaline phosphatase (U/L) & 0.073 & 0.021 & 11.713 & 0.001 & 1.075 & $1.032-1.121$ \\
Lipase (U/L) & 2.485 & 0.736 & 11.400 & 0.001 & 12.000 & $2.836-50.775$ \\
Lactate (mmol/L) & 2.603 & 0.733 & 12.614 & 0.001 & 13.500 & $3.210-56.770$ \\
Nitric oxide (U/mL) & 2.079 & 0.612 & 11.531 & 0.001 & 8.000 & $2.409-26.567$ \\
Cardiac troponin I (ng/mL) & 1.910 & 0.536 & 12.703 & 0.001 & 6.750 & $2.362-19.290$ \\
\hline
\end{tabular}

Table 8. Multivariate logistic regression model of the analysis of the activity of both alkaline phosphatase and lipase, and the level of lactate, nitric oxide, and cardiac troponin $I$ in peritoneal fluid in donkeys with obstructive colic.

\begin{tabular}{lcccccc}
\hline Variables & B & S.E. & Wald & P & OR & 95 \% CI \\
\hline Alkaline phosphatase (U/L) & -3.196 & 1.578 & 4.101 & 0.034 & 0.041 & $0.002-0.902$ \\
Lipase (U/L) & -2.888 & 1.468 & 3.872 & 0.030 & 0.056 & $0.003-0.988$ \\
Lactate (mmol/L) & -3.519 & 1.484 & 5.624 & 0.018 & 0.030 & $0.002-0.543$ \\
Constant & 4.958 & 1.571 & 9.961 & 0.002 & & \\
\hline
\end{tabular}

\section{DISCUSSION}

Obstructive colic is a major disease state that causes severe abdominal pain and a frequent and important cause of death in horses and donkeys. These species are prone to colic compared to others due to their unique anatomy of digestive system. Under the field condition, markers of ischemic bowel are useful for predicting, monitoring, and managing the obstructive colic in equine. To improve the diagnostic accuracy while examining a horse with colic, several studies regarding clinical and laboratory examination are needed. Therefore, the current study aimed to assess the cellular profile and certain biochemical parameters of the peritoneal fluid, highlighting their diagnostic sensitivity and specificity as biomarkers for early diagnosis of intestinal ischemia in donkeys (Equus asinus) as a model of equine.

In the existing study, peritoneal fluid analysis had remarkable cellular alterations as a consequence of intestinal strangulation. These findings were similar to those reported by Hanson and Albanese (2015). As a consequence of strangulating obstruction, the red blood cells migrate across the bowel wall into the peritoneal fluid, followed by white blood cells. Thus, the earliest sensitive indicator of intestinal strangulating lesion is the change in the color of peritoneal fluid from straw to red color. The recorded increase in the total nucleated cells count in the peritoneal fluid is mostly related to the increase in the segmented neutrophils, which was observed in the donkeys under investigation. Constable et al. (2017) stated that the total leukocyte count was ranged between 10,000 and 150,000 cell $/ \mu \mathrm{L}$ in horses with peritonitis and/or intestinal infarction. Furthermore, the results of sensitivity and specificity of the cellular profile of the peritoneal fluid demonstrated its usefulness as an aid in diagnosing intestinal ischemia in the studied donkeys as previously reported elsewhere (Faleiros et al., 2011; Duesterdieck-Zellmer et al., 2014).

With respect to the ultrasonographic imaging results, the intestinal peristalsis was absent, the intestine appeared as several distended loops adjacent to each other with a thickened and edematous wall, and the amount of the fluid in the cranial abdomen increased. These findings were similar to those reported by le Jeune and Whitcomb (2014) and Constable et al. (2017). The 
results of ultrasound imaging provided a diagnostic aid for evaluating horses with colic and assisting in the differentiation of medical from surgical colic.

Alkaline phosphatase activity in the peritoneal fluid increased significantly in donkeys with intestinal obstruction compared to the control group. Such increase may be attributed to the increased hepatic exposure to endotoxin, inflammatory mediators, enteric bacteria, and stretched bile ducts as a result of ileus. Consequently, measurement of alkaline phosphatase activity in the peritoneal fluid aids in diagnosis of more severe intestinal lesions requiring surgery in horses. These findings were similar to those previously reported (Saulez et al., 2004).

Lipase activity in the peritoneal fluid increased significantly in donkeys with intestinal ischemia compared to the control group. The elevated lipase activity suggests acute pancreatic injury secondary to both intestinal obstruction and shock associating acute abdominal disorders (Grulke et al., 2003; Talbot et al., 2011). In human studies, it has been reported that shock can lead to hypo-perfusion of the splanchnic area with a resultant multi-organ dysfunction, including pancreas (Venneman et al., 1993). The pancreas is mostly vulnerable to hypovolemia due to both its specific structure and the nature of the blood distribution within it (Endrich et al., 1990).

Lactate level in the peritoneal fluid was significantly greater in donkeys with intestinal obstruction compared to those in the control group. Horses suffering colic are in imminent shock and incurring an oxygen deficit. In oxygen-deficient states, hypoxic tissues undergo anaerobic glycolysis with the end result being an increased lactic acid production. Lactate accumulates in the cells and enters the circulation exacerbating metabolic acidosis (Rogatzki et al., 2015). Consequently, the high lactate level is well correlated with greater degree of bowl damage and ischemia as the horses succumbed to diffuse peritonitis 3 days later (Peloso and Cohen, 2012). Several studies discussed the clinical importance and prognostic significance of preoperative assessment of lactate in peritoneal fluid in horses with signs of colic and intestinal ischemia (Delesalle et al., 2007; Tennent-Brown et al., 2010). In a study conducted on adult horses with large colon volvulus, the serum lactate level at admission in nonsurvived horses was significantly increased compared with those of survivors. Moreover, the serum lactate level returned to its normal level in the survivors, but continued to be higher in non-survivors (Johnston et al., 2007).

The gut is considered an instigator for systemic inflammation. In the present study, intestinal obstruction and ischemia, and gut disuse may prime the body for a hyper-active inflammatory response, including increased gut inflammation and breakdown of gut barrier function, thus disturbing the intestinal microbiomes and predisposing animal to significant inflammation again (Annane, 2005). The outcome is a deleterious vasodilatation and capillary leakage, with concomitant hypovolemia and increased metabolic demand. As a result, when oxygen delivery is insufficient to meet metabolic demand, tissue hypoxia develops giving rise to production of lactate as a by-product of anaerobic cellular respiration (Ebrahim, 2011). The cellular hypoxia is confounded due to impaired cellular oxygen extraction, which is mediated at a mitochondrial level through nitric oxide, blocking the mitochondrial electron transfer chain with subsequent increased mitochondrial derived reactive oxygen species concentration (Jeffrey Man et al., 2014).

In the existing study, there was a significant increase in the level of nitric oxide in the peritoneal fluid obtained from donkeys with obstructive colic compared to the control group. Mirza et al. (1999) found that nitric oxide level in the peritoneal fluid was significantly increased subsequent to small intestinal strangulating obstruction. Nitric oxide exerts either cytotoxic or cytoprotective effects during intestinal inflammation, including ischemia-reperfusion injury, depending upon the source, duration, magnitude, and timing of its production (Mirza et al., 2005; Granger and Kvietys, 2015). Hypoxemia and ischemia occurring as a result of intestinal strangulating volvulus cause trans-mural colonic damage with disruption of the mucosal barrier subsequent to ischemia and secondary to reperfusion injury. Reperfusion injury is usually initiated by production of oxygen free radicals and perpetuated by neutrophils and inflammatory mediators (Granger and Kvietys, 2015). The constitutive nitric oxide protects the intestinal mucosal barrier, while the inducible isoform of nitric oxide is usually expressed in numerous cells, in response to cytokines or another stimulus, leading to sustained release of large quantities of nitric oxide (Beckman and Crow, 1993; Granger and Kvietys, 2015). The produced nitric oxide reacts with superoxide radicals to produce peroxynitrite, causing appreciable tissue damage (Nakao et al., 2009).

Cardiac troponin I level increased greatly in the peritoneal fluid collected from the studied donkeys with obstructive colic compared to control group, suggesting myocardial injury as a result of intestinal ischemia. Up to now, there are no available published data regarding the potential significance of measuring cardiac troponin $I$ in the peritoneal fluid of equine with obstructive colic. An increase in the level of cardiac troponin I at admission was significantly associated with occurrence of ventricular arrhythmias and the outcome is surgical treatment (Radcliffe et al., 2012; Díaz et al., 2014). Furthermore, Nath et al. (2012) reported that the serum cardiac troponin I level was significantly elevated in $36 \%$ of horses suffered from strangulating lesions and $47 \%$ of horses with inflammatory lesions. The cardiovascular system is sensitive to the effect of vasoactive substances, 
including nitric oxide produced during systemic inflammatory response. Nitric oxide is a vocative mediator responsible for the reduced myocardial contractility during systemic inflammatory response syndrome. Likewise, endotoxin and pro-inflammatory cytokines have been shown to induce myocardial depression, which is probably mediated through nitric oxide (Bohlen, 2015).

Conclusion: The results herein demonstrated that the increased total nucleated cells count, the increased activity of both alkaline phosphatase and lipase, and increased level of lactate are sensitive and specific predictors to assist in the early diagnosis of intestinal obstruction in donkeys (Equus asinus) with obstructive colic. Thus, it provides evidence about the ability of the assessment of cellular profile and measurement of such biochemical parameters in the peritoneal fluid to provide a diagnostic plan for early diagnosis as well as for detection of the deleterious consequences of intestinal ischemia in donkeys (Equus asinus) with an intestinal obstructive lesion. Also, along with its excellent predictive power, the peritoneal fluid is easy to obtain, making it a practical tool of potentially widespread applicability. These non-invasive and promising tools may offer opportunities for early diagnosis of the intestinal ischemia in equine with intestinal obstruction and potentially more rational choice to differentiate between surgically and conservatively treated colic in such animals.

Conflict of interest: None of the authors of this paper has a financial or personal relationship with other people or organizations that could inappropriately influence or bias the content of the paper.

\section{REFERENCES}

Annane, D., E. Bellissant, and J. M. Cavaillon, (2005). Septic shock - Review. Lancet. 365(9453): 6378.

Archer, D. C., and C. J. Proudman, (2006). Epidemiological clues to preventing colic. Vet. J. 172(1):29-39.

Beckman, J. S., and J. P. Crow, (1993). Pathological implications of nitric oxide, superoxide, and peroxynitrite formation. Biochem. Soc. Trans. 21(2): 330-334.

Beckman, J. S., and W. H. Koppenol, (1996). Nitric oxide, superoxide, and peroxynitrite: The good, the bad, and the ugly. Am. J. Physiol. 271(5 Pt 1): C1424-C1437.

Bohlen, H. G., (2015). Nitric oxide and the cardiovascular system. Compr. Physiol. 5(2): 808-823.

Constable, P. D., K. W. Hinchcliff, S. H. Done, and W. Grünberg, (2017). Abdominocentesis for peritoneal fluid. In: Veterinary Medicine: A textbook of the diseases of cattle, horses, sheep, pigs and goats. $11^{\text {th }}$ Edition, Volume 1. Diseases of the Alimentary Tract: Non-ruminant. Missouri, United States: Elsevier Ltd. P. 186 190.

Curtis, L., I. Trewin, G. England, J. Burford, and S. Freeman, (2015). Veterinary practitioners' selection of diagnostic tests for the primary evaluation of colic in the horse. Vet. Rec. Open. 2(2): e000145.

De Backer, D., (2003). Lactic acidosis. Intensive Care Medicine 29(5): 699-702.

Delesalle, C., J. Dewulf, R. Lefebvre, J. Schuurkes, J. Proot, L. Lefere, and P. Deprez, (2007). Determination of Lactate Concentrations in Blood Plasma and Peritoneal Fluid in Horses with Colic by an Accusport Analyzer. J. Vet. Intern. Med. 21 (2): 293-301.

Díaz, O. M., M. M. Durando, E. K. Birks, and V. B. Reef, (2014). Cardiac troponin I concentrations in horses with colic. J. Am. Vet. Med. Assoc. 245(1): 118-125.

Duesterdieck-Zellmer, K. F., J. H. Riehl, E. C. McKenzie, A. M. Firshman, M. E. Payton, and M. E. Gorman, (2014). Effects of abdominocentesis technique on peritoneal fluid and clinical variables in horses. Equine Vet. Educ. 26(5): 262-268.

Ebrahim, G. J., (2011). Sepsis, septic shock and the systemic inflammatory response syndrome. J. Trop. Pediatr. 57(2): 77-79.

Endrich, B., E. Klar, and F. Hammersen, (1990). The microcirculation of the pancreas: State of the art. In: Gastrointestinal Microcirculation, Edition. Messmer K, Hammersen F. Basel, Switzerland: Karger Publishers. p. 144-174.

Faleiros, R. R., D. G. Macoris, C. H. C. Saquetti, A. C. Aita, A. Farias, E. B. Malheiros, and I. B. M. Sampaio, (2011). Peritoneal fluid changes in horses subjected to small colon distension. Pesqui. Vet. Brasil. 31(5): 367-373.

Gomaa, N. A., H. M. M. Ibrahim, M. Ishii, M. N. Nassif, and S. A. El-khodery, (2013). Dose-Dependent Effects of Mosapride Citrate on Duodenal and Cecal Motility in Donkeys (Equus asinus). Int. J. Vet. Sci. Med. 1: 51-56.

Granger, D. N., and P. R. Kvietys, (2015). Reperfusion injury and reactive oxygen species: The evolution of a concept. Redox. Biol. 6: 524-551.

Grulke, S., G. Deby Dupont, D. Cassart, M. Gangl, I. Caudron, M. Lamy, and D. Serteyn, (2003). Pancreatic Injury in Equine Acute Abdomen Evaluated by Plasma Trypsin Activity and Histopathology of Pancreatic Tissue. Vet. Pathol. 40(1): 8-13. 
Hameed, A. M., V. W. Lam, and H. C. Pleass, (2015). Significant elevations of serum lipase not caused by pancreatitis: a systematic review. HPB (Oxford). 17(2): 99-112.

Hanson, R. R., and V. Albanese, (2015). Gastrointestinal Disease. In Robinson's Current Therapy in Equine Medicine. $7^{\text {th }}$ Edition. Chapter 75 Small Intestine Colic. Missouri, United States: Elsevier. P. 328-332.

Hanson, R., A. Nixon, R. Gronwall, D. Meyer, and J. Pendergast, (1992). Evaluation of peritoneal fluid following intestinal resection and anastomosis in horses. Am. J. Vet. Res. 53(2): 216-221.

Hardy, J., (2004). Critical care. In: Reed S.M., Bayly W.M., Sellon D.C. Equine Internal Medicine 2nd Edition, Elsevier, USA. p. 273-288.

Ibrahim, H. M. M., N. A. Gomaa, A. El- Mahmoudy, S. A. El-khodery, M. Ishii, and M. N. Nassif, (2013). Ultrasonographic evaluation of duodenal and cecal motility after administration of different doses of domperidone in donkeys (Equus Asinus). J. Vet. Adv. 3(6): 179-187.

Jeffrey Man, H. S., A. K. Tsui, and P. A. Marsden, (2014). Nitric oxide and hypoxia signaling. Vitam. Horm. 96: 161-192.

Johnston, K., S. J. Holcombe, and J. G. Hauptman, (2007). Plasma lactate as a predictor of colonic viability and survival after 3601 volvulus of the ascending colon in horses. Vet. Surg. 36(6): 563-567.

Juzwiak, J. S., C. A. Ragle, C. M. Brown, J. D. Krehbiel, and R. F. Slocombe, (1991). The effect of repeated abdominocentesis on peritoneal fluid constituents in the horse. Vet. Res. Commun. 15(3): 177-180.

Kanwar S., B. L. Tepperman, D. Payne, L. R. Sutherland, and P. Kubes, (1994). Time course of nitric oxide production and epithelial dysfunction during ischemia/reperfusion of the feline small intestine. Circ. Shock. 42: 135-140.

Kaya, G., and C. Iben, (2009). Diagnostic value of serum alkaline phosphatase activity and serum bile acids and causative effect of specific bacteria in faeces of colic horses. Wien. Tierärztl. Mschr. Vet. Med. Austria 96: 195-202.

Kubes, P., (1993). Ischemia-reperfusion in feline small intestine: a role for nitric oxide. Am. J. Physiol. 264 (1 Pt 1): G143-G149.

Kubes, P., J. Hunter, and D. N. Granger, (1992). Ischemia/reperfusion-induced feline intestinal dysfunction: Importance of granulocyte recruitment. Gastroenterol. 103(3): 807-812.

Latson, K., J. Nieto, P. Beldomenico, and J. Snyder, (2005). Evaluation of peritoneal fluid lactate as a marker of intestinal ischaemia in equine colic Equine Vet. J. 37(4): 342-346.

le Jeune, S., and M. B. Whitcomb, (2014). Ultrasound of the equine acute abdomen. Vet. Clin. North Am. Equine Pract. 30(2): 353-381.

Liao, X. P., Y. X. She, and M. Li, (1995). Changes in body fluid markers in intestinal ischemia. J. Ped. Surg. 30(10): 1412-1415.

Lott, J. A., and C. J. Lu, (1991). Lipase isoforms and amylase isoenzymes: assays and application in the diagnosis of acute pancreatitis. Clin. Chem. 37(3): 361-368.

Mirza, M. H., T. L. Seahorn, J. L. Oliver, G. Hosgood, and R. M. Moore, (2005). Detection and comparison of nitric oxide in clinically healthy horses and those with naturally acquired strangulating large colon volvulus. Can. J. Vet. Res. 69(2): 106-115.

Mirza, M., J. L. Oliver, T. L. Seahorn, G. Hosgood, and R. M. Moore, (1999). Detection and comparison of nitric oxide in clinically normal horses and those with naturally acquired small intestinal strangulation obstruction. Can. J. Vet. Res. 63(4): 230-232.

Moncada, S., and A. Higgs, (1993). The L-arginine-nitric oxide pathway. N. Engl. J. Med. 329(27): 2002 -2012 .

Nakao, A., R. Sugimoto, T. R. Billiar, and K. R. McCurry, (2009). Therapeutic antioxidant medical gas. J. Clin. Biochem. Nutr. 44(1): 113.

Nath, L. C., G. A. Anderson, and K. W. Hinchcliff, (2012). Clinicopathologic evidence of myocardial injury in horses with acute abdominal disease. J. Am. Vet. Med. Assoc. 241(9): 1202-1208.

Peloso, J. G., and N. D. Cohen, (2012). Use of serial measurements of peritoneal fluid lactate concentration to identify strangulating intestinal lesions in referred horses with signs of colic. J. Am. Vet. Med. Assoc. 240(10): 1208-1217.

Radcliffe, R. M., T. Divers, D. J. Fletcher, H. Mohammed, and M. S. Kraus, (2012). Evaluation of L-lactate and cardiac troponin I in horses undergoing emergency abdominal surgery. J. Vet. Emerg. Crit. Care. 22 (3): 313 319.

Rogatzki, M. J., B. S. Ferguson, M. L. Goodwin, and L. B. Gladden, (2015). Lactate is always the end product of glycolysis. Front Neurosci. 9(Article 22): 1-7.

Saulez, M. N., C. K. Cebra, and S. Tornquist, (2004). The diagnostic and prognostic value of alkaline phosphatase activity in serum and peritoneal fluid from horses with acute colic. J. Vet. Intern. Med. 18(4): 564-567. 
Talbot, W., W. Kipar, G. Edwards, and E. Singer, (2011). Acute necrotising pancreatitis in three ponies. Tierarztl. Prax. Ausg. G. Grosstiere Nutztiere. 39(6): 393-396.

Tennent-Brown, B., P. Wilkins, S. Lindborg, G. Russell, and R. Boston, (2010). Sequential Plasma Lactate Concentrations as Prognostic Indicators in Adult Equine Emergencies. J. Vet. Intern. Med. 24 (1): 198-205.

Tietz, N. W., and D. F. Shuey, (1993). Lipase in serum the elusive enzyme: an overview. Clin. Chem. 39(5): 746-756.

Tinker, M. K., N. A. White, P. Lessard, C. D. Thatcher, K. D. Pelzer, B. Davis, and D. K. Carmel, (1997). Prospective study of equine colic incidence and mortality. Equine Vet. J. 29(6): 448-453.
Van Hoogmoed, L., L. D. Rodger, S. J. Spier, I. A. Gardner, T. B. Yarbrough, and J. R. Snyder, (1999). Evaluation of peritoneal fluid $\mathrm{pH}$, glucose concentration, and lactate dehydrogenase activity for detection of septic peritonitis in horses. J. Am. Vet. Med. Assoc. 214(7): 1032-1036.

Venneman, I., G. Deby-Dupont, and M. Lamy, (1993). Pancreatic cellular injury after cardiopulmonary bypass. In: Yearbook of Intensive Care and Emergency Medicine. Edition. Berlin Heidelberg New York: Springer-Verlag. p. 297308.

Yamout, S. Z., J. E. Nieto, P. M. Beldomenico, J. E. Dechant, S. leJeune, and J. R. Snyder, (2011). Peritoneal and plasma D-lactate concentrations in horses with colic. Vet. Surg. 40(7): 817-824. 